\title{
EATING VLBI and KVN-Yebes observations of AGN jets
}

Bong Won Sohn ${ }^{1,2,7 *}$, Gabriele Giovannini
Kazuhiro Hada ${ }^{3,9}$, Marcello Giroletti $^{3}$, Motoki Kino ${ }^{5}$,

Orienti $^{3}$, Mareki Honma ${ }^{6,9}$, Hiroshi Nagai ${ }^{9}, 10$, Tomoaki Oyama ${ }^{6}$, Rocco Lico $^{3}$, Sejin Oh $^{1}$, Guangyao Zhao ${ }^{1}$, Pietro Cassaro ${ }^{11}$, Alessandro Orfei ${ }^{3}$, Mateo Stagni ${ }^{3}$, Taehyun Jung $^{1,2}$, Pablo Vincente ${ }^{12}$, Maria Rioja ${ }^{13,14}$, and Richard Dodson ${ }^{13}$

${ }^{1}$ Korea Astronomy and Space Science Institute (KASI), Daedeokdae-ro 776, Yuseong-gu, 34055

Daejeon, Korea E-mail: bwsohn@kasi.re.kr

${ }^{2}$ University of Science and Technology, Gajeong-ro 217, Yuseong-gu, 34113 Daejeon, Korea

${ }^{3}$ INAF Istituto di Radioastronomia, Via Gobetti 101, 40129 Bologna, Italy

${ }^{4}$ Dipartimento di Fisica e Astronomia, Universita di Bologna, Via Gobetti 93/2, 40129 Bologna, Italy

${ }^{5}$ Kogakuin University, Academic Support Center, 2665-1 Nakano, Hachioji, Tokyo 192-0015, Japan

${ }^{6}$ Mizusawa VLBI Observatory, National Astronomical Observatory of Japan, 2-21-1 Owasa, Mitaka, Tokyo 181-8588, Japan

${ }^{7}$ Yonsei University, Yonsei-ro 50, Seodaemun-gu, 03722 Seoul, Korea

${ }^{8}$ Institute of Astronomy \& Astrophysics, Academia Sinica, P.O. Box 23-141, Taipei 10617,

Taiwan

${ }^{9}$ Department of Astronomical Science, The Graduate University for Advanced Studies

(SKENDAI), 2-21-1 Osawa, Mitaka, Tokyo 181-8588, Japan

${ }^{10}$ National Astronomical Observatory of Japan, Osawa 2-21-1, Mitaka, Tokyo 181-8588, Japan

${ }^{11}$ INAF Stazione Radioastronomica di Noto Ctr.da Renna Bassa C.P. 161, 96017 Noto, Italy

${ }^{12}$ Observatorio Astronomico Nacional Instituto Geografico Nacional, Apartado de Correos 112,

28803 Alcala de Henares, Spain

${ }^{13}$ ICRAR, M468, The University of Western Australia, 35 Stirling Hwy, Crawley, Western

Australia 6009, Australia

${ }^{13}$ Observatorio Astronomico Nacional (IGN), Alfonso XII, 3 y 5, 28014 Madrid, Spain

Firstly, we introduce 'EATING VLBI', East Asia To Italy: Nearly Global VLBI. It is an Eurasian VLBI collaboration of East Asia (EAVN) and Italy (VLBIT). The cooperation of the VLBIT and the EAVN provided high angular resolution to the sensitive EAVN. We report the preliminary EATING VLBI images of four AGNs. Secondly, we introduce KVN-Yebes joint VLBI observation program which aims high precision VLBI astrometry. Finally, KaVA (KVN and VERA Array) polarimetry results is briefly reported. With VLBIT and Yebes, KaVA/EAVN will provide a powerful VLBI polarimetry opportunity in the near future.

14th European VLBI Network Symposium \& Users Meeting (EVN 2018)

8-11 October 2018

Granada, Spain 


\section{EATING VLBI}

Firstly, we introduce 'EATING VLBI', East Asia To Italy: Nearly Global VLBI. It is an Eurasian VLBI collaboration of East Asian VLBI Network (EAVN; An, Sohn \& Imai (2018) and Hada et al.(2019)) and the Italian VLBI facilities (VLBIT). The cooperation of the VLBIT and the EAVN provided the high angular resolution to the sensitive EAVN. Here we report the EATING observations of four AGNs with 3C84, Mrk501, TXS0506+056 and FSRQ 1633+382 (Figure 1). These AGNs are characterized by presence of resolved jets with limb-brightened structures or with recent high energy alerts. Here we present the preliminary results of the first epoch on 3rd February 2018. We are conducting multi-epoch observations of these sources. EATING VLBI is operational at $22 \mathrm{GHz}$ with bandwidth of $256 \mathrm{MHz}(16 \mathrm{MHz}$ x $16 \mathrm{IF}$ or $32 \mathrm{MHz}$ x $8 \mathrm{IF})$ and will be operational at $43 \mathrm{GHz}$ soon. Further extension of frequency and polarization capability will follow. Proposals should be submitted to VLBIT (9th October) and EAVN (1st November) separately, next call in six months. An MOU for coordinated Call for Proposal is in discussion. Below are the preliminary images of four AGNs observed with 8 EATING VLBI telescopes ( $3 \mathrm{KVN}, 4$ VERA and Medicina) on 3rd February 2018. We are monitoring these sources with three to four months interval. Integration time of each source: TXS0506 $220 \mathrm{~min} .$, 3C84 $260 \mathrm{~min}$., Mrk501 $340 \mathrm{~min}$., and FSRQ1633 170 min. In April 2019, EATING VLBI workshop will be held in Bologna.

\section{KVN-Yebes Astrometry}

We launched KVN-Yebes joint Source-Frequency-Phase-Referencing (SFPR; Rioja et al. 2015) observation program in order to study AGN core motions. FSRQ 1633+382 $(4 \mathrm{C}+38.41)$ has shown rich structural evolution of the jet (Ro et al. 2019). Some of the explanations for the complicated evolution of the jet include the core movement, e.g. due to opacity variation, jet viewing angle change (precession/nutation), or binary SMBH. In order to measure the core position change, we
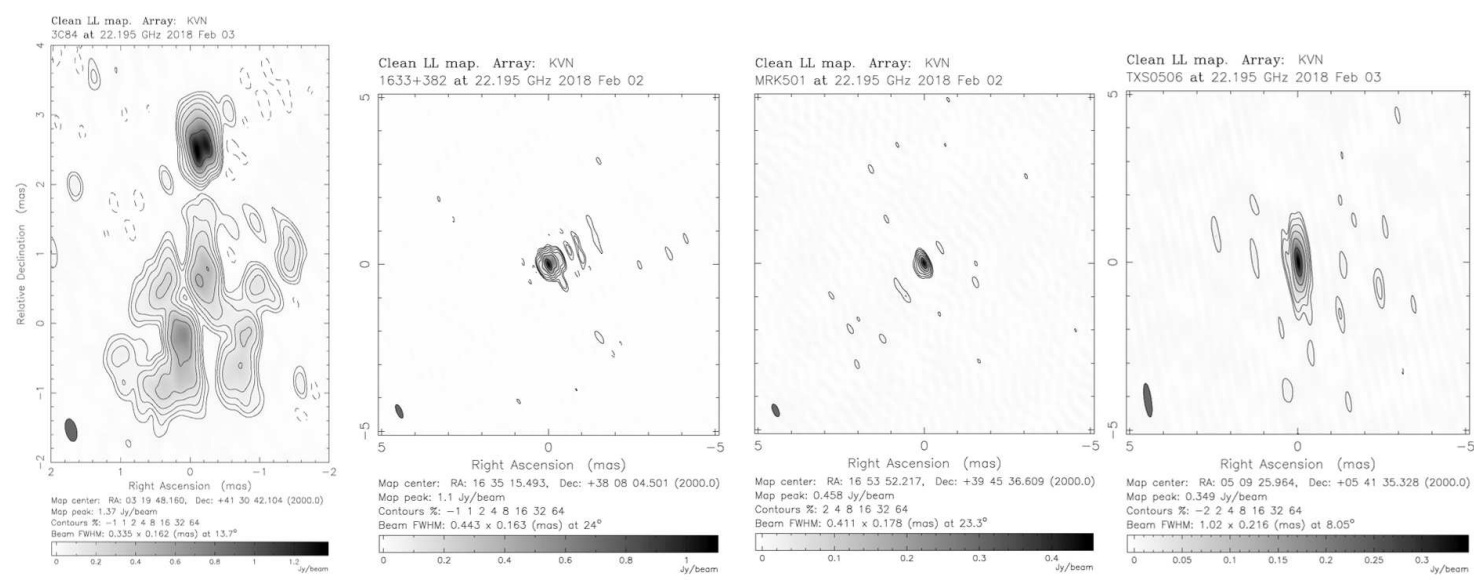

Figure 1: From left to right, EATING VLBI $22 \mathrm{GHz}$ images of 3C84, FSRQ 1633+382 (4C+38.41), Mrk 501 and TXS 0506+056 are presented.

\footnotetext{
* Speaker.

${ }^{\dagger}$ A footnote may follow.
} 
launched KVN-Yebes SFPR astrometric VLBI program. Simultaneous multi frequency observation capability of $\mathrm{KVN}$-Yebes is essential to estimate position change and therefore opacity effect or others accurately. Recently we added 3C66A/B pair to the program (see Figure 2), which is debated as a binary SMBH candidate (Sudou et al. 2003; Zhao et al. 2015). Based on the coming results, we are going to propose an AGN large program of KVN-Yebes SFPR astrometry.

\section{KaVA polarimetry}

We present KaVA polarization map of 3C84 ( $22 \mathrm{GHz}, 128 \mathrm{MHz}$ bandwidth, full polarization) obtained on 24th February 2018 with 3 KVN and 2 VERA telescopes (Figure 2). The Eastern edge of the source shows polarized structure which needs further inspection. KaVA polarization observation is in commissioning phase. With VLBIT and Yebes, KaVA/EAVN will provide a powerful VLBI polarimetry opportunity in the near future.

\section{References}

[1] T. An, B. W. Sohn, \& H. Imai, 2018, Nature Astronomy, 118, 2 [arXiv: 1802.01136 ].

[2] H. Hada, B. W. Sohn, M. Kino, G. Giovannini et al. 2019, in proceedings of 14th EVN symposium, EVN2018.

[3] M. Rioja, R. Dodson, T. Jung, B. W. Sohn, 2015, AJ,202, 150.

[4] H. Ro, B. W. Sohn, A. Chung, T. Krichbaum, 2019, in proceedings of 14th EVN symposium, EVN2018.

[5] H. Sudou, S. Iguchi, Y. Murata, Y. Taniguchi, 2003, Science,1263, 300.

[6] G. Zhao, Y. Chen, Z. Shen, H. Sudou, S. Iguchi, 2015, AJ,149, 46.
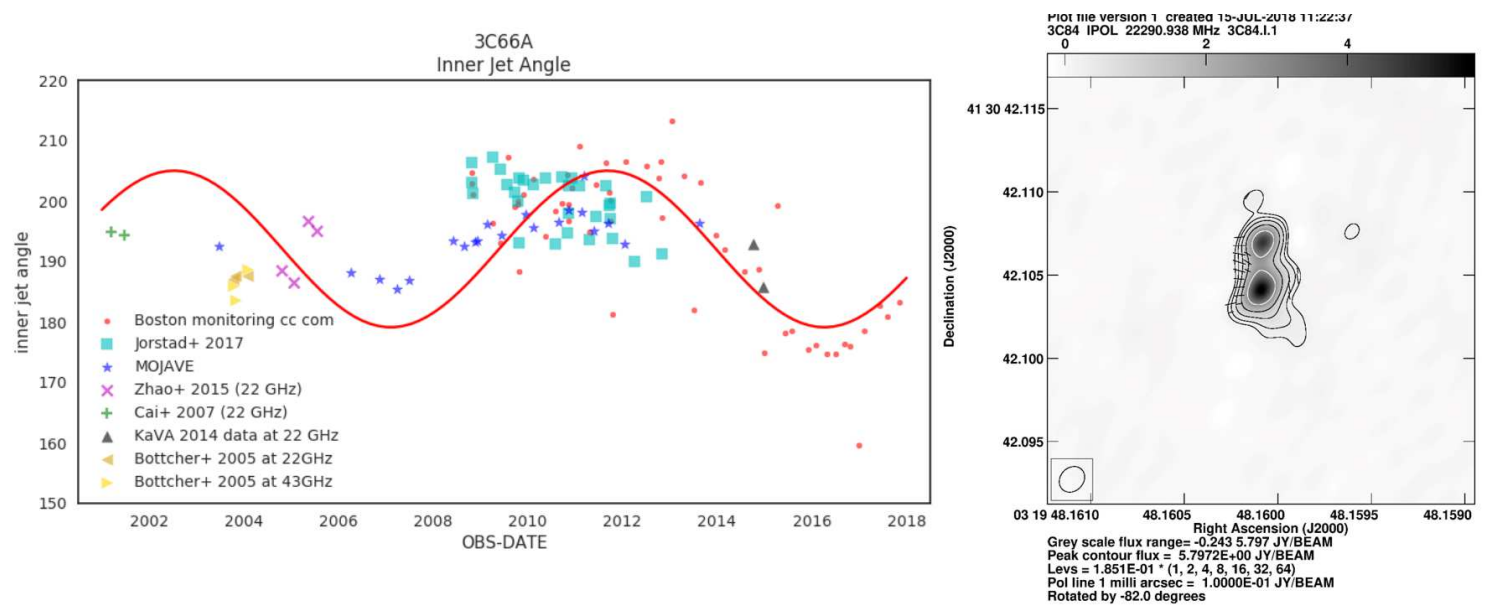

Figure 2: [Left]Long-term jet position angle change of 3C66A (by J. Kim and G. Zhao). [Right] Preliminary result of KaVA polarization observation on $3 \mathrm{C} 84$. 